\title{
60
}

\section{Defining a Family of Feedback Signals for Multimedia Communication Devices}

\author{
Cynthia A. Sikora and Linda A. Roberts \\ Lucent Technologies: Bell Labs Innovations \\ 200 Laurel Avenue, 4E-508 \\ Middletown, New Jersey 07748 \\ USA \\ Tel: (908) 957-6135 \\ Email: csikora@lucent.com and lin@lucent.com
}

\begin{abstract}
A family of auditory feedback signals was created for multimedia business devices. This family was derived from a wide variety of options, including real world, musical and communications sounds. In Experiment I, typical business users mapped 48 sounds to functions and rated the pleasantness and appropriateness of the sounds. Experiment II tested the best sounds for each function (based on the findings of Experiment I) within the context of a graphical user interface business communications simulation. Users rated the pleasantness and appropriateness of each sound and selected the best sound option for each function. Experiment III evaluated the resultant family of auditory signals for discriminability using similarity ratings. Results showed that, real world sounds mapped most reliably to the functions; users consistently preferred musical sounds to the real world sounds. Of the eleven feedback signals selected for specific functions, four were communications based and seven were musical. None of the real world auditory signals were acceptable. The resulting family of sounds was generally discriminable with the exception of one pair. The apparent dimensions used to make judgments about the sound pairings were duration, average pitch and harmonic complexity.
\end{abstract}

KEYWORDS Auditory Feedback, Auditory Icons, Earcons, Sound, User Interface Design

Human-Computer Interaction: INTERACT'97 S. Howard, J. Hammond \& G. Lindgaard (editors)

Published by Chapman \& Hall OIFIP 1997 


\section{INTRODUCTION}

In today's market, the use of sound as feedback has become more possible with advances in technology and the widespread availability of computer equipment. However, auditory feedback is still lacking in many communications products supporting multimedia and graphical user interfaces. The emphasis in most products has been on visual feedback provided to the user, which is not always sufficient for communications tasks. However, as multimedia applications become viable, there has been an increasing interest in developing auditory signals that can ease the complexity of the user's interaction with the applications. The introduction of these new multimedia devices and services also provides an opportunity to include signature "look" and "listen" elements.

During the past decade, two classes of sounds have been proposed as auditory feedback signals (Brewster, Wright and Edwards, 1993). The first, auditory icons, use environmental sounds that have a strong semantic link with the object represented by the sound. An example of this may be the sound of a door closing when a file is closed. The use of these "real world" sounds has been advocated due to the discernible functional mappings inherent in these sounds (Blattner, Sumikawa and Greenberg, 1989; Gaver, 1986; Mynatt, 1994). The second class of sounds, earcons (Brewster et. al., 1993), refer to abstract synthesized sounds. Implicit in this latter category are the sounds that are more musical in origin, with well-defined pitches, rhythms, harmonies and timbres. Jones and Turner (1989) showed that, whereas subjects preferred earcons, they were better able to associate auditory icons to functions.

One issue regarding the implementation of real world sounds concerns users' reactions to listening to them repeatedly over time in an actual business application. One of our concerns is that users may perceive the real world sounds as annoying and trite. More abstract, musical sounds may be less intrusive, providing appropriate feedback without being distracting to the user or others.

Abstract sounds (i.e., earcons) that may provide a successful alternative to real world sounds include communications and musical sounds. We refer to communications sounds as relatively simple signals derived from traditional electronic tones used in telecommunications products. We refer to musical sounds as more complex abstract musical signals that are unconstrained by currently used telecommunications audible feedback tones.

Table 1: Auditory signals were sought for eleven functions which are labeled and described.

\begin{tabular}{|c|c|}
\hline FUNCTION & DESCRIPTION \\
\hline Confirmation & $\begin{array}{l}\text { Sound associated with } \\
\text { performing a task correctly. }\end{array}$ \\
\hline $\begin{array}{l}\text { Distinctive } \\
\text { Ring }\end{array}$ & $\begin{array}{l}\text { Ringing that can be associated } \\
\text { with a particular type of call. }\end{array}$ \\
\hline $\begin{array}{l}\text { Drop from } \\
\text { Conference }\end{array}$ & $\begin{array}{l}\text { Auditory feedback to indicate } \\
\text { that an individual has left a } \\
\text { conference call in progress. }\end{array}$ \\
\hline End Call & $\begin{array}{l}\text { Auditory feedback that a call } \\
\text { has been terminated. }\end{array}$ \\
\hline Error & $\begin{array}{l}\text { Auditory indication that an error } \\
\text { condition or incorrect action } \\
\text { has occurred. }\end{array}$ \\
\hline $\begin{array}{c}\text { Hold } \\
\text { Reminder }\end{array}$ & $\begin{array}{l}\text { Alerting signal that a call is } \\
\text { placed, or remains, on hold. }\end{array}$ \\
\hline $\begin{array}{c}\text { Join } \\
\text { Conference }\end{array}$ & $\begin{array}{l}\text { Sound indicating that a new } \\
\text { individual has joined a } \\
\text { conference call. }\end{array}$ \\
\hline $\log 0$ & $\begin{array}{l}\text { Auditory signal associated with } \\
\text { the start up of a product. }\end{array}$ \\
\hline $\begin{array}{l}\text { Personalized } \\
\text { Ring }\end{array}$ & $\begin{array}{l}\text { A selectable ring used to } \\
\text { distinguish one user's phone } \\
\text { from others. }\end{array}$ \\
\hline Send Call & $\begin{array}{l}\text { Auditory indicator provided for } \\
\text { buffered dialing to confirm that } \\
\text { the digit string has been sent. }\end{array}$ \\
\hline $\begin{array}{l}\text { Status } \\
\text { Indicator }\end{array}$ & $\begin{array}{l}\text { Auditory signal indicating wait } \\
\text { time during system activity. }\end{array}$ \\
\hline
\end{tabular}

A professional sound designer/musician designed both communications and musical feedback signals for eleven different communications functions (shown in Table 1). The musician was asked to create one communication and one musical sound for each function, but he created many more alternatives than requested (due to his 
enthusiasm for the project). Real world signals for the functions were selected from sound libraries.

In Experiment 1, we investigated which feedback signals (i.e., real world, communications, and musical) mapped best to the eleven functions. In addition, we asked subjects to evaluate the sound's pleasantness and its appropriateness for use in a business setting. Based on the results of this experiment, we derived three candidate sounds for each function. In Experiment 2, we tested the pleasantness and appropriateness of the candidate set of sounds within the context of a simulated business communications application. Results of the second study enabled us to then select the single best feedback sound for each of the eleven functions. The primary goal of Experiment 3 was to ensure sufficient distinctiveness within the resultant family of 11 sounds. In addition, we sought to determine which dimensions of sound users evaluated when listening to the signals in order to guide future research in this area.

\section{EXPERIMENT I: SOUND EXPLORATION}

The experiment was designed to find any natural mapping of the sounds to the functions. The sounds were also evaluated for their overall pleasantness, and their appropriateness for a business environment. Thirty-eight computer literate office workers participated as paid subjects. The subjects were tested in four groups of approximately 10 per group.

The 48 sounds that were evaluated consisted of 22 musical sounds, 14 communications oriented sounds and 12 real world sounds. The 12 real world sounds were selected from an initial set of 30 real world sounds. The ideas for the 30 real world sounds were generated using brainstorming techniques with a small group of college students who volunteered to participate during their internship at our company (Lucent Technologies, formerly AT\&T). The real world sound ideas were then investigated for availability from sound libraries. Due to difficulty in recognizing some of the sounds, the real world sounds were limited to those that provided an adequate auditory representation of the referent. Examples of real world sounds and the intended functions include footsteps (wait status indicator), paper crumpling (error), door opening (join conference), door closing (drop from conference), knocking on door (hold reminder), phone hanging up (end call) and applause (confirmation).

Each group of subjects was provided with a study packet. After filling out a brief demographic questionnaire, subjects were given verbal instructions about the task. Subjects were also encouraged to read the written version of the instructions provided in the study packet. The subjects were told that they would hear sounds and be asked to select the function that best matched the sound as a feedback signal and to rate the sound for pleasantness, appropriateness and confidence of the function chosen. The instructions emphasized that the sounds were intended for a state-of-the-art business application. The subjects were also requested to assume that all telephone activity was presented in a multimedia application on a personal computer.

The subjects were provided with a sheet with definitions of the 11 communications functions that require auditory feedback. Subjects were instructed to read through the list of functions and their definitions and to ask questions about anything that was unclear. The function description list was available to the subjects throughout the study. Definitions for the constructs being rated were also provided:

- Functional Mapping refers to the function that is best represented by a given sound.

- Pleasantness refers to the pleasing quality of the sound.

- Appropriateness refers to how well the sound is suited for a business application.

- Confidence refers to their degree of confidence that the sound provides a good match with a function.

The subjects were instructed that they would hear each sound twice and that they should listen very closely. Subjects were advised that any given sound could be modified to accommodate the needs of a specific function (e.g., repeated for ringing or lower volume for hold reminder). They were also invited to write in any comments they might have. 
The subjects were given two practice trials, and any questions they had were addressed. Each group of subjects was then presented the 48 sounds, in a different random order. Each sound was played twice. After each sound was presented, the subjects indicated which of the 11 functions the sound best mapped to, and then rated the sound for its overall pleasantness, appropriateness in a business setting, and the confidence they had in selecting that function for that sound. For each of the three latter tasks, a 9-point scale was used where 9 denoted "very pleasant", "very appropriate" and "very confident". After an intermediate task, the groups were presented with the sounds again in a different randomized order and made the same mapping and rating judgments.

The data were analyzed to determine which sounds mapped most often to the functions. Overall, the real world sounds mapped most predictably to the functions. The communications sounds mapped to the functions with reasonable consistency. The musical sounds mapped least consistently to the functions.

The results for the pleasantness ratings are shown in Figure 1. There was a reliable main effect of time $[F(1,36)=7.36, p=.01]$, demonstrating that listeners gave higher ratings upon the second hearing of them. There was also a reliable effect of sound class $[F(2,72)=252.55$, $\mathrm{p}<.01]$. In this case, the musical and communications sounds received significantly higher pleasantness ratings relative to the real world sounds. There were no significant interactions.

Ratings of the appropriateness for a business environment are shown in Figure 2. As occurred for the pleasantness ratings, there were main effects of time $[F(1,36)=5.26$, $p=.027]$ and of sound class $[F(2,72)=168.62, p<.01]$ and no interactions. Communications and musical sounds were judged to be reliably more appropriate for a business setting than were the real world sounds.

Based on these results, we derived three auditory signals for each function for further evaluation in Experiment II. Eleven real world sounds, 7 communications sounds and 15 musical sounds were selected. The eleven real world sounds each mapped very well to their respective functions, but achieved very low ratings for pleasantness
Figure 1: Pleasantness ratings for the classes of audibles are shown. Ratings are based on a nine point scale where 9 is very pleasant and I is very unpleasant.

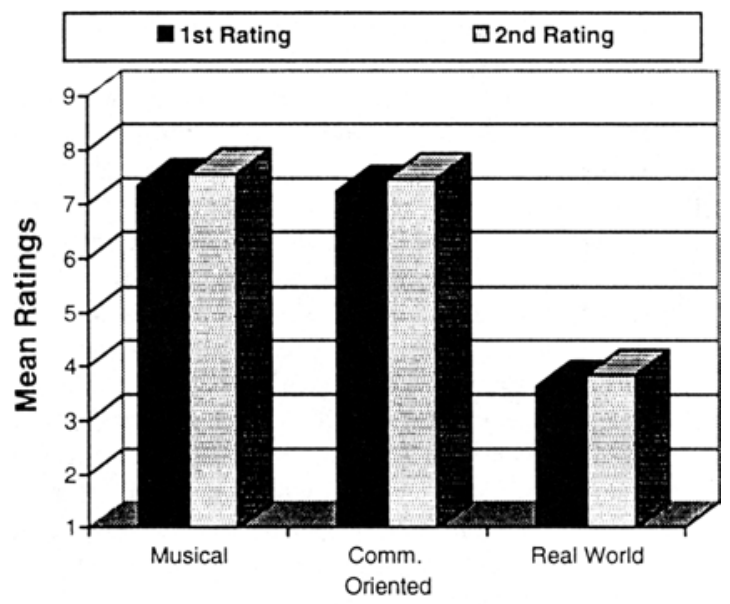

Sound Families

Figure 2: Appropriateness ratings for the classes of audibles are shown. Ratings are based on a nine point scale where 9 is very appropriate and $l$ is very inappropriate for a business environment.

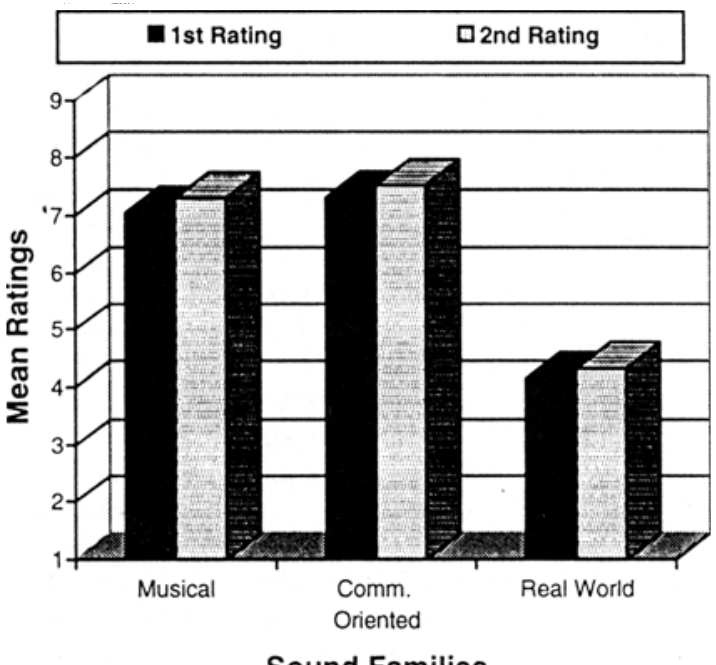

Sound Families 
and appropriateness. The remaining two candidates for each function were those musical or communications sounds that had the best mapping. When no clear mappings to functions were evident (e.g., two or more sounds mapped relatively well to a given function), sounds were selected that had the highest pleasantness and appropriateness ratings.

\section{EXPERIMENT II: EVALUATING SOUNDS IN A BUSINESS CONTEXT}

The subset of sounds selected from Experiment I was based primarily on functional mapping. Experiment II was designed to test the sounds further within the context of a simulated business communications PC application. Twenty-two computer literate office workers who were not tested in the previous study participated as paid subjects. The subjects were tested in groups of two to four.

Each group of subjects was given a study packet which included written instructions, questionnaires and rating sheets. The business application simulation was used to present the sounds in a business scenario within a graphical telecommunications application. Subjects were told that they would be rating auditory feedback sounds for pleasantness and appropriateness within a business application. They were also instructed that, within a trial, they would select the one sound that they felt was best for that function. As in Experiment I, it was emphasized that the sounds should be envisioned for a multimedia telephone in a state-of-the-art office environment. Subjects were told that they would only hear each sound once within the scenario before being asked to make the ratings. The constructs being rated (i.e., pleasantness and appropriateness) were defined prior to the task.

Projected from an LCD projector attached to the PC, subjects were shown a simulation that provided a context and scenario for each of the functions. Subjects began with a practice scenario with three different sounds for a function not included in the study. After making individual ratings and then selecting the best sound for the function, any questions were answered before continuing. Before each scenario, the function being depicted was defined. The scenario was shown three times with three different sounds, presented in different orders for each group. After hearing each sound, the subjects rated its pleasantness and appropriateness on a 9 point scale. The subjects then heard all three sounds in the scenario again before selecting the sound that they would most prefer for that function.

Figure 3 shows the mean ratings for pleasantness and appropriateness of the sounds included in Experiment II. There was a significant difference among the sound families for both the pleasantness $[F(2,702)=46.23$, $p<.01]$ and appropriateness $[F(2,702)=28.89, p<.01]$ ratings of the sounds included in Experiment II. Overall musical sounds received the highest ratings for both pleasantness and appropriateness. In both cases real world sounds received the lowest ratings.

Figure 3: The mean pleasantness and appropriateness ratings of the auditory signals included in Experiment II are shown based on a nine point scale where 9 reflects a positive response.

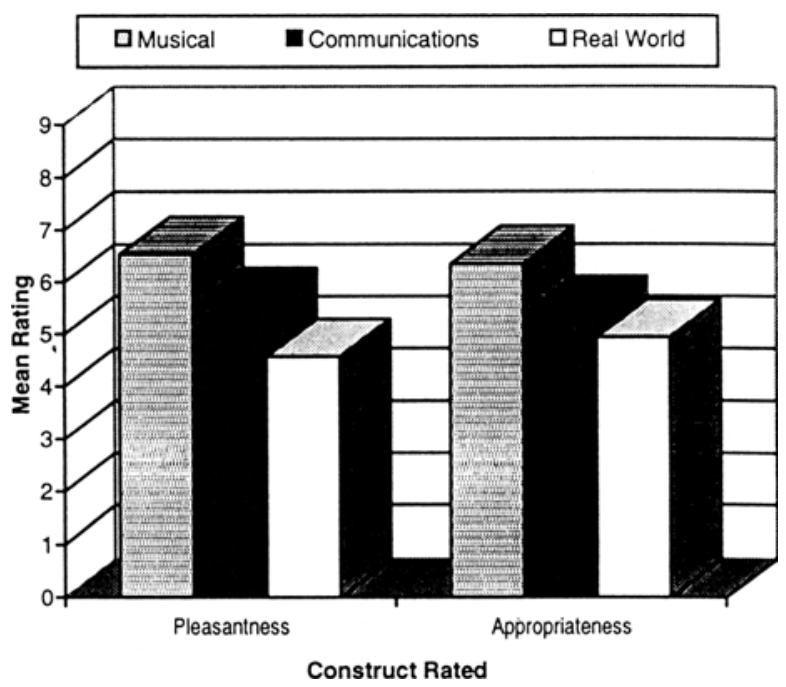

As occurred in Experiment I, the real world sounds were consistently rated poorly on pleasantness and appropriateness. In addition, subjects seldom chose a real world sound as the best choice for any of the functions. 
The musical sounds had consistently high pleasantness and appropriateness ratings and were most frequently selected as the best choice. Communications sounds varied in subjective ratings and occasionally proved to be the best selection for a given function. None of the real world sounds were viable choices due to low ratings.

Figure 4 shows the mean pleasantness and appropriateness ratings for the sounds derived based on best choice judgments. Although all sounds selected were rated positively for pleasantness and appropriateness there was still a significant difference between the musical and communications sound families. There was a main effect of sound class for both pleasantness $[F(1,236)=30.13, p<.01]$ and appropriateness $[F(1,236)$ $=11.35, \mathrm{p}<.01]$. Musical sounds were rated more highly on both constructs than communications oriented sounds.

Figure 4: The mean ratings of pleasantness and appropriateness for the auditory signals chosen as best for the functions are shown, based on a nine point scale.

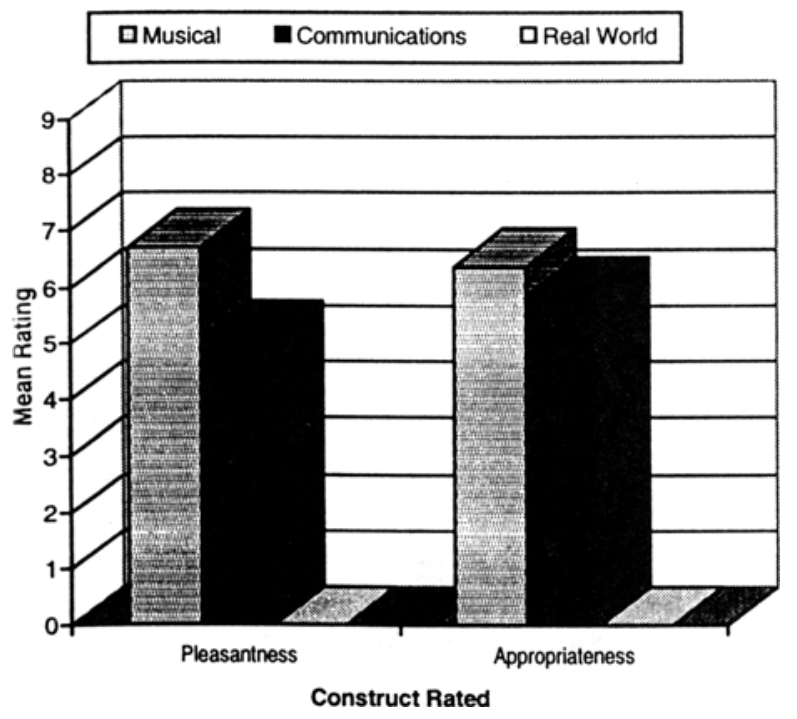

Eleven sounds, one for each function, were subsequently selected for use as auditory feedback. The selections were based primarily on the frequency of the sounds being selected as "best" for that function. Rating data for pleasantness and appropriateness was used to verify that the selected sounds were considered pleasant and appropriate within the business application task. Seven of the sounds were musical sounds and four were communications sounds. None of the real world sounds was selected.

\section{EXPERIMENT III: EVALUATING THE DISCRIMINABILITY OF THE RESULTANT FAMILY OF SOUNDS}

The final sound selections for the eleven functions requiring auditory feedback were chosen based on the preference data from subjects in Experiment II. Although each sound selected mapped adequately to the function and was rated highly for overall pleasantness and appropriateness in a business environment, the question of discriminability within the family still existed. Experiment III was designed to evaluate the discriminability of the eleven sounds within the sound family identified.

Subjects rated the similarity of the eleven sounds and described the dimensions they used to make their judgments. Thirty-eight computer literate office workers, who were not involved in either of the previous studies, participated in the study and were compensated for their time. The subjects understood that the sounds being evaluated were intended to provide auditory feedback in a business application, but the eleven functions were not described to them.

Subjects filled out a brief demographic questionnaire and were then given verbal and written instructions describing the task. The subjects were tested individually using a computer simulation. The task required judging the degree of similarity of each pairing of the 11 sounds using a five point scale, where 5 denoted "very similar" and 1 denoted "very dissimilar". Subjects were given a brief introduction to the task and then completed the task at their own pace.

To listen to a pair of sounds, the subject clicked on each of two buttons to play the sounds. The subject was able to play each sound as many times as necessary prior to making a rating. Subjects indicated perceived similarity by clicking one of the buttons on the five point scale. 
After each rating, the subject clicked on the "next" button to indicate their readiness for the next pair of sounds. There were 55 sound pairings of the 11 sounds. Subjects completed a questionnaire which inquired about the strategies used for making the similarity ratings.

The data from the sound similarity ratings revealed that the discriminability within the sound family was quite good with the exception of one pair of sounds. The sound for the Error and End Call functions were judged to be very similar.

Based on subjective reporting in conjunction with a multidimensional scaling analysis of the data, three dimensions appear to be used by the subjects to make their similarity judgments. The dimensions are best described as duration, average pitch and harmonic complexity of the sounds. The complexity of the sound was described by subjects using various terms, but in each case it seemed to reflect the degree to which the sound was simple or complex. The two sounds that were judged to be too similar to be easily discriminated were both short, low pitched simple sounds.

\section{DISCUSSION}

An investigation of functional mapping revealed that, for real world sounds, subjects generally selected the function for which the sound had been intended. The communications sounds, whose abstract musical tones were based on learned pairings, had less consistent functional mappings. The musical sounds, which had no previous learned association, were more randomly mapped to the functions.

To further investigate which sounds should be selected for use, the subjective ratings of the sounds were analyzed within the context of a business application. The more musically oriented sounds were most preferred. Clearly, our results support earlier findings (Jones and Furner, 1989) that the type of auditory feedback most preferred by subjects is not the same as those sounds that provided the best functional mapping. Rather, the users' reactions indicate that real world signals are less appropriate for actual applications relative to abstract musical feedback. These results emphasize the need to solicit judgments in addition to functional mappings when choosing appropriate audible feedback signals. Indeed, our results demonstrate that subjective ratings of pleasantness and appropriateness are more reliable selection criteria.

Once individual sounds were identified for each function and the preferences for the sounds were verified, the discriminability of the sounds as a family was investigated. Overall, the sounds were easily discriminable. An exception was that the auditory feedback for Error was rated as being very similar to End Call. The sound for the Error function was replaced as a result of this data.

Discriminability data provided a glimpse at the strategy used to make similarity judgments about auditory feedback signals. Although described in various terms, pitch, duration and harmonic complexity seem to be critical, observable aspects of sounds. In fact, the two sounds that were rated as very similar were similar on all three dimensions. These results do not, however, preclude the importance of other aspects of sound since our results may simply be a reflection of our particular sound stimulus set.

In summary, results of these experiments indicate that real world sounds are less appropriate for business communications applications than are more abstract musically based sounds. Considering the richness and complexity of the graphically based products users have available today, it is a natural progression for the accompanying auditory feedback to rise to a similar level of sophistication and appeal.

These conclusions must, however, be treated with some caution since there were several flaws in our methodology. First, there were more musical sounds in the stimulus sets in Experiment I than communications and real world sounds and subjects may have assumed an experimenter bias when making their judgments. Second, the real world sounds may have been disadvantaged since they were selected from sound libraries and were not privy to the same creativity and technical manipulation as were the communications and musical sounds. However, we have recently carried out research that addresses these limitations in the present studies and preliminary results demonstrate patterns that 
mirror the current results. Another concern is that, since the more musical signals are not as intuitively mapped to the functions relative to real world sounds, there may be issues relative to end user training particularly for visually impaired users. The long term use of these sounds may also be questioned. For example, it may be that abstract sounds become concrete enough to be perceived as annoying at some point. The study was also limited to testing subjects in the United States. These sounds are being tested in other countries to ensure they are appropriate and pleasant to people in other parts of the world. At this point, these sounds have been tested in Singapore, Turkey and India. Those results are currently being compared to equivalent testing done in the United States.

\section{ACKNOWLEDGMENTS}

The authors would like to thank Mark Barasch of Mark Barasch Music Productions LTD for his creative musical contributions, La Tondra Murray and Andrea D'Amico for running subjects and Dan Teibel for his technical input and support.

\section{REFERENCES}

Blattner, M., Sumikawa, D. \& Greenberg, R. (1989). Earcons and icons: Their structure and common design principles. Human-Computer Interaction, 4, 11-44. Brewster, S., Wright, P. \& Edwards, A. (1993). An Evaluation of Earcons for Use in Auditory HumanComputer Interfaces, in Proc. INTERCHI'93 Human Factors in Computing Systems (Amsterdam, April 2429, 1993), ACM Press, pp. 222-227.

Gaver, W. (1986). Auditory icons: Using sound in computer interfaces. Human-Computer Interaction, 2 , 167-177.

Jones, S. \& Furner, S. (1989). The constructions of audio icons and information cues for human-computer dialogues. Contemporary Ergonomics, Proceedings of the Ergonomics Society's 1989 Annual Conference, T. Megaw (Ed.)

Mynatt, E. (1994). Designing with Auditory Icons: How Well do We Identify Auditory Cues?, in Proc. CHI'94 Human Factors in Computing Systems (Boston, April 24-28, 1994), ACM Press, pp. 269-270. 